\title{
The Langevin Limit of the Nosé-Hoover-Langevin Thermostat
}

\author{
Jason Frank • Georg A. Gottwald
}

Received: 30 November 2010 / Accepted: 13 April 2011 / Published online: 30 April 2011

(C) Springer Science+Business Media, LLC 2011

\begin{abstract}
In this note we study the asymptotic limit of large variance in a stochastically perturbed thermostat model, the Nosé-Hoover-Langevin device. We show that in this limit, the model reduces to a Langevin equation with one-dimensional Wiener process, and that the perturbation is in the direction of the conjugate momentum vector. Numerical experiments with a double well potential corroborate the asymptotic analysis.
\end{abstract}

Keywords Thermostat methods · Nosé-Hoover dynamics · Langevin dynamics · Homogenization methods $\cdot$ Canonical sampling

\section{Introduction}

Deterministic thermostats for canonical sampling were introduced by Nosé $[16,17]$ and Hoover [6] and are commonly used in molecular dynamics to simulate systems at constant temperature. These methods extend the phase space of a Hamiltonian system by one or more degrees of freedom such that the extended dynamics - when projected back onto the original phase space-preserve the Gibbs distribution, i.e. the canonical distribution is a steady state of the Liouville flow associated with the projected dynamics. Bulgac and Kusnezov [1] generalized the Hoover thermostat to noncanonical Hamiltonian systems. Proving ergodicity of deterministic thermostats has presented a challenge (see for example [13, 14]), and this has led recently to the construction of stochastically forced thermostat equations, referred to here as Nosé-Hoover-Langevin (NHL) thermostats, in which the augmented degrees of freedom satisfy a Langevin equation $[11,20]$.

\footnotetext{
J. Frank ( $\varangle)$

Centrum Wiskunde \& Informatica, P.O. Box 94079, 1090 GB Amsterdam, The Netherlands e-mail: jason@cwi.nl

G.A. Gottwald

School of Mathematics and Statistics, University of Sydney, Sydney, NSW 2006, Australia

e-mail: georg.gottwald@sydney.edu.au
} 
Consider a Hamiltonian system

$$
\frac{d x}{d t}=\mathbb{J} \nabla H(x),
$$

where $x \in \mathbb{R}^{n}, \mathbb{J}^{T}=-\mathbb{J}$ and $H: \mathbb{R}^{n} \rightarrow \mathbb{R}$. Assuming that the system is in thermal equilibrium with an external heat bath of constant temperature $T$, the NHL thermostated dynamics is given by

$$
\begin{aligned}
& d x=[\mathbb{\nabla} H(x)+y g(x)] d t \\
& d y=\left[\frac{1}{\alpha}(\nabla \cdot g(x)-\beta \nabla H \cdot g(x))-\frac{\alpha \sigma^{2}}{2} y\right] d t+\sigma d W,
\end{aligned}
$$

with $y \in \mathbb{R}$ and initial conditions $x(0)=x_{0}$ and $y(0)=y_{0}$. Here $W$ is a one-dimensional independent Brownian motion, and $\beta=1 /\left(k_{B} T\right)$ is proportional to the inverse temperature with $k_{B}$ being the Boltzmann constant. We denote by $\nabla$ the gradient operator with respect to the $x$-variables only. The NHL system (2) is constructed to preserve the extended measure $\rho(x, y)=\exp \left(-\beta H(x)-\alpha y^{2} / 2\right)$ and differs from a classical Langevin equation in a number of ways. For one the Wiener process $W$ does not directly perturb the dynamics in $x$, but instead does so indirectly through $y$ after integration. In essence this can be seen as providing a memory effect; that is, the perturbed dynamics in $x$ is non-Markovian. Furthermore, (2) makes use of only a scalar stochastic variable, and it is a question how this scalar perturbation pervades the rest of the dynamics. To assure ergodicity, the (otherwise arbitrary) vector field $g(x)$ can be chosen to satisfy a so-called Hörmander controllability condition [15]. The Hörmander condition for the coupled system (2) can be formulated by writing it in the form of a stochastic differential equation with degenerate noise term $d z=r(z) d t+s(z) d W$, where $r$ and $s$ are vector fields in $\mathbb{R}^{n+1}$ and $W(t)$ is a scalar Wiener process. The Hörmander condition states that the Lie algebra generated by the vector fields $r$ and $s$ spans the whole $\mathbb{R}^{n+1}$, and therefore ensures that the noise eventually pervades all dimensions of phase space. In the present case, this reduces to the requirement that the Lie algebra generated by vector fields $\mathbb{J} \nabla H$ and $g$ spans $\mathbb{R}^{n}$, see [11].

In this paper, we are interested in the limiting case when the thermostat variable $y$ can have large values due to an increased variance $1 / \alpha$. In a heuristic way, it can be understood that the limiting dynamics is a Langevin equation (see below) as the thermostat variable $y$ will tend to Brownian motion in the case when its relaxation time $1 /\left(\alpha \sigma^{2}\right)$ tends to zero. We will in the following make this heuristic more precise. In particular we will see that additional drift terms arise.

A general Langevin thermostat for (1) is

$$
d x=\left[\mathbb{J} \nabla H(x)+\frac{1}{2} \nabla \cdot\left(\Sigma(x) \Sigma^{T}(x)\right)-\frac{\beta}{2} \Sigma(x) \Sigma^{T}(x) \nabla H(x)\right] d t+\Sigma(x) d w,
$$

where $w(t)$ is a Wiener process in $\mathbb{R}^{n}$ and $\Sigma(x) \in \mathbb{R}^{n \times n}$ induces multiplicative noise. The form of this equation is dictated by the demand that the Gibbs measure $\rho=\exp (-\beta H(x))$ be stationary under the flow of the associated Fokker-Planck equation, for arbitrary choice of $\Sigma(x)$.

We show that the limiting dynamics of the NHL model (2) is a Langevin model (3) with rank-one multiplicative noise $\Sigma(x)$. For mechanical systems with $x=(q, p)$ and separable Hamiltonian $H=\frac{1}{2} p^{T} M^{-1} p+V(q)$, we will see that in the large noise limit the NHL model (2) is equivalent to the momentum directed Langevin thermostat proposed in [12], 
of which the stochastic velocity rescaling thermostat of Bussi et al. [2, 3] is a particular case.

We introduce the scaling

$$
\alpha \rightarrow \epsilon^{2} \alpha, \quad y \rightarrow \frac{1}{\epsilon} y, \quad \sigma \rightarrow \frac{1}{\epsilon^{2}} \sigma
$$

with $\epsilon \ll 1$. The NHL equation (2) then becomes

$$
\begin{aligned}
& d x=\left[\mathbb{J} \nabla H(x)+\frac{1}{\epsilon} y g(x)\right] d t \\
& d y=\left[\frac{1}{\epsilon} \frac{1}{\alpha}(\nabla \cdot g(x)-\beta \nabla H \cdot g(x))-\frac{1}{\epsilon^{2}} \frac{\alpha \sigma^{2}}{2} y\right] d t+\frac{1}{\epsilon} \sigma d W .
\end{aligned}
$$

This particular scaling in $\epsilon$ allows for the effective reduced dynamics to capture diffusive effects rather than classical averaging.

\section{Homogenization}

We will analyze the NHL system (5) in the framework of the backward Kolmogorov equation for the conditional expectation value of some sufficiently smooth observable $\phi(x, y)$ defined as

$$
v\left(x_{0}, y_{0}, t\right)=\mathbb{E}\left[\phi(x(t), y(t)) \mid x(0)=x_{0}, y(0)=y_{0}\right] .
$$

Here the expectation value is taken with respect to Brownian motion driving paths. Dropping the subscripts, we study the following Cauchy problem for $t \in[0, \infty)$

$$
\begin{aligned}
\frac{\partial v}{\partial t}(x, y, t) & =\mathscr{L} v(x, y, t) \\
v(x, y, 0) & =\phi(x, y),
\end{aligned}
$$

with the generator

$$
\mathscr{L}=\frac{1}{\epsilon^{2}} \mathscr{L}_{0}+\frac{1}{\epsilon} \mathscr{L}_{1}+\mathscr{L}_{2}
$$

where

$$
\begin{aligned}
& \mathscr{L}_{0}=-\frac{\alpha \sigma^{2}}{2} y \partial_{y}+\frac{1}{2} \sigma^{2} \partial_{y y} \\
& \mathscr{L}_{1}=y g(x) \cdot \nabla+\frac{1}{\alpha}(\nabla \cdot g(x)-\beta \nabla H \cdot g(x)) \partial_{y} \\
& \mathscr{L}_{2}=\mathbb{J} \nabla H \cdot \nabla .
\end{aligned}
$$

Pioneered by Khasminsky [7], Kurtz [8-10] and Papanicolaou [18] singular perturbation theory can be formulated for a perturbation expansion according to

$$
v(x, y, t)=v_{0}+\epsilon v_{1}+\epsilon^{2} v_{2}+\cdots .
$$


A comprehensive exposition of the theory of stochastic model reductions and their implementation is given for example in $[19,21]$. Substituting the series (10) into the backward Kolmogorov equation (6) we obtain at lowest order, $\mathscr{O}\left(1 / \epsilon^{2}\right)$,

$$
\mathscr{L}_{0} v_{0}=0
$$

The fast dynamics associated with the generator $\mathscr{L}_{0}$ is given by an Ornstein-Uhlenbeck process and is therefore ergodic. ${ }^{1}$ Ergodicity of the fast process implies that expectation values do not depend on initial conditions $y$. Hence the constant solution

$$
v_{0}=v_{0}(x, t)
$$

is the only solution of (11).

Ergodicity is equivalent to the existence of a unique invariant density, i.e. that the equation

$$
\mathscr{L}_{0}^{\star} \rho=0,
$$

has a unique solution $\rho_{\infty}(y)$. Here $\mathscr{L}_{0}^{\star}$ is the formal $L_{2}$-adjoint of the generator $\mathscr{L}_{0}$. For later reference we present the unique invariant density of the fast Ornstein-Uhlenbeck process associated with $\mathscr{L}_{0}^{\star}$

$$
\rho_{\infty}(y)=\sqrt{\frac{\alpha}{2 \pi}} \exp \left(-\frac{\alpha}{2} y^{2}\right) .
$$

At the next order, $\mathscr{O}(1 / \epsilon)$, we obtain

$$
\mathscr{L}_{0} v_{1}=-\mathscr{L}_{1} v_{0}
$$

To assure boundedness of $v_{1}$ (and thereby of the asymptotic expansion (10)) the solvability condition prescribed by the Fredholm alternative has to be satisfied. Equation (13) is solvable only provided the right-hand side is in the space orthogonal to the (one-dimensional) null space of the adjoint $\mathscr{L}_{0}^{\star}$, i.e. if

$$
\left\langle\mathscr{L}_{1} v_{0}\right\rangle_{\rho_{\infty}}=-\langle y g(x)\rangle_{\rho_{\infty}} \cdot \nabla v_{0}(x, t)=0,
$$

where $\langle h\rangle_{\rho_{\infty}}:=\int \rho_{\infty}(y) h(x, y) d y$ denotes the average of an observable $h(x, y)$ over the invariant density. Since $\langle y\rangle_{\rho_{\infty}}=0$, there exists a solution of (13), which is readily calculated as

$$
v_{1}(x, y, t)=\frac{2}{\alpha \sigma^{2}} y g \cdot \nabla v_{0}+R(x),
$$

where $R(x)$ lies in the kernel of $\mathscr{L}_{0}$. Note that the vanishing of the average of the thermostat perturbation with respect to the invariant measure induced by the fast thermostat variable (i.e. $\langle y g\rangle_{\rho_{\infty}}=0$ ) implies that classical averaging would produce a trivial reduced dynamics with $d x=\mathbb{J} \nabla H d t$ without any trace of the fast stochastic thermostat variable.

At the next order, $\mathscr{O}(1)$, we obtain the desired evolution equation for $v_{0}$,

$$
\mathscr{L}_{0} v_{2}=\frac{\partial}{\partial t} v_{0}-\mathscr{L}_{1} v_{1}-\mathscr{L}_{2} v_{0}
$$

\footnotetext{
${ }^{1}$ As discussed above, the Ornstein-Uhlenbeck process was introduced precisely into the framework of thermostat equations to render the full system ergodic.
} 
Again a solvability condition has to be imposed which reads

$$
\frac{\partial}{\partial t} v_{0}=\left\langle\mathscr{L}_{2} v_{0}\right\rangle_{\rho_{\infty}}+\left\langle\mathscr{L}_{1} v_{1}(x, t)\right\rangle_{\rho_{\infty}}
$$

Using $\left\langle y^{2}\right\rangle_{\rho_{\infty}}=1 / \alpha$ we obtain the full reduced slow backward-Kolmogorov equation

$$
\frac{\partial}{\partial t} v_{0}=\left(\mathbb{J} \nabla H+\frac{2}{\alpha^{2} \sigma^{2}}\left[\nabla \cdot\left(g g^{T}\right)-\beta g g^{T} \nabla H\right]\right) \cdot \nabla v_{0}+\frac{2}{\alpha^{2} \sigma^{2}} g g^{T}: \nabla \nabla v_{0} .
$$

Here we define that the divergence operator acting on matrix valued functions $h$ acts by contraction as $\{\nabla \cdot h\}_{i}=\partial_{j}\left(h_{i j}\right)$, and the inner product of matrices is defined as

$$
A: B=a_{i j} b_{i j}=\operatorname{Tr}\left(A B^{T}\right),
$$

which induces the Frobenius norm.

Note that $R(x)$ does not contribute to the dynamics. We can therefore choose $R(x)=0$ in order to assure that $\langle v\rangle_{\rho_{\infty}}=v_{0}+\mathcal{O}\left(\epsilon^{2}\right)$.

The slow reduced Langevin equation associated with the reduced backward Kolmogorov equation (17) is then

$$
d X=F(X) d t+S(X) d W \quad \text { with } X(0)=x_{0},
$$

with one-dimensional Wiener process $W$ and where the drift coefficient vector $F(X)$ and the diffusion coefficient vector $S(X)$ are given by

$$
\begin{aligned}
& F(X)=\mathbb{J} \nabla H+\frac{2}{\alpha^{2} \sigma^{2}}\left[\nabla \cdot\left(g g^{T}\right)-\beta g g^{T} \nabla H\right] \\
& S(X)=\frac{2}{\alpha \sigma} g .
\end{aligned}
$$

We conclude that (18)-(19) is in the form of the Langevin equation (3) for the case $\Sigma(x)=$ $\frac{2}{\alpha \sigma} g(x)$ and scalar Wiener process $W$.

\section{Reduced Model for Molecular Dynamics}

The original thermostat devices of Nosé and Hoover $[6,16,17]$ were developed for molecular dynamics problems in canonical Hamiltonian form. (For an application of the NHL thermostat to point vortices, see [4].) Since Nosé-Hoover thermostats are primarily used in the molecular dynamics context, in this section we specify the reduced Langevin model derived in the previous section to the particular case of mechanical systems. To this end we take $n=2 d$, and consider a mechanical system with positive diagonal mass matrix $M$ and phase space coordinates $x=(q, p), q, p \in \mathbb{R}^{d}$, and with Hamiltonian

$$
H(x)=H(q, p)=\frac{1}{2} p^{T} M^{-1} p+V(q),
$$

and canonical structure matrix $\mathbb{J}=\left(\begin{array}{cc}0 & I \\ -I & 0\end{array}\right)$. The deterministic equations of motion (1) are then given by

$$
\frac{d q}{d t}=M^{-1} p, \quad \frac{d p}{d t}=-\frac{\partial}{\partial q} V(q)
$$


For the NHL system (2) we make the specific choice $g(x)=g(q, p)=\left(0, p^{T}\right)^{T}$. Note that the Hörmander condition is immediate here, since the original vector field $M^{-1} p,-\partial V / \partial q$ and the perturbation vector field $g(q, p)=(0, p)$ are trivially linearly independent. In molecular dynamics, this choice is physically motivated, since the thermostat acts to slow or accelerate the motion. One would then need to check the Hörmander condition for the given potential $V$. Our numerical example in the next section is in $\mathbb{R}^{2}$, so the Hörmander

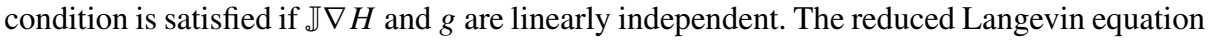
(18)-(19) then takes the form

$$
\begin{aligned}
& d q=M^{-1} p d t \\
& d p=\left[-\nabla_{q} V(q)+\frac{2}{\alpha^{2} \sigma^{2}}\left(d+1-\beta p^{T} M^{-1} p\right) p\right] d t+\frac{2}{\alpha \sigma} p d W,
\end{aligned}
$$

from which it is apparent that the noise and dissipation act in the direction of the generalized momentum vector $p$. In particular, we note that the reduced Langevin equation (21) and the NHL system (2) preserve the set of equilibria of the full deterministic dynamics (1). We also point out that the reduced Langevin equation (21) is equivalent to the momentum directed Langevin thermostat described in [12] as a generalization of the stochastic velocity rescaling thermostat of Bussi et al. [2, 3].

\section{Numerical Verification}

In this section we will numerically illustrate that the reduced Langevin equation (18) is a good pathwise model of the small- $\epsilon$ limit of the NHL model (5). We show this for a simple system with one degree of freedom $(d=1)$. Parameters for the NHL model (2) and its reduced Langevin equation (21) are chosen to be $\alpha=\epsilon^{2}, \sigma=1 / \epsilon^{2}$.

Both the NHL model (2) and the reduced model (21) were implemented using a splitting method, equivalent to the Störmer-Verlet method for the Hamiltonian vector field $\mathbb{J} \nabla H$, and solving the thermostat dynamics using a composition of a half-step each of the split-step backward Euler method [5] and the Euler-Maruyama method, which effects the midpoint rule in the absence of the noise term. For the reduced model (21) this becomes

$$
\begin{aligned}
q_{n+1 / 2} & =q^{n}+\frac{\Delta t}{2} M^{-1} p_{n} \\
p_{n+1 / 2}^{\prime} & =p_{n}-\frac{\Delta t}{2} \nabla_{q} V\left(q_{n+1 / 2}\right) \\
p_{n+1 / 2}^{\prime \prime} & =p_{n+1 / 2}^{\prime}-\frac{\Delta t}{2} \Gamma\left(p_{n+1 / 2}^{\prime \prime}\right) \\
p_{n+1 / 2} & =p_{n+1 / 2}^{\prime \prime}+\frac{2}{\alpha \sigma} p_{n+1 / 2}^{\prime \prime}\left(W_{n+1 / 2}-W_{n}\right) \\
p_{n+1}^{\prime} & =p_{n+1 / 2}-\frac{\Delta t}{2} \Gamma\left(p_{n+1 / 2}\right)+\frac{2}{\alpha \sigma} p_{n+1 / 2}\left(W_{n+1}-W_{n+1 / 2}\right) \\
p_{n+1} & =p_{n+1}^{\prime}-\frac{\Delta t}{2} \nabla_{q} V\left(q^{n+1 / 2}\right) \\
q_{n+1} & =q_{n+1 / 2}+\frac{\Delta t}{2} M^{-1} p_{n+1},
\end{aligned}
$$



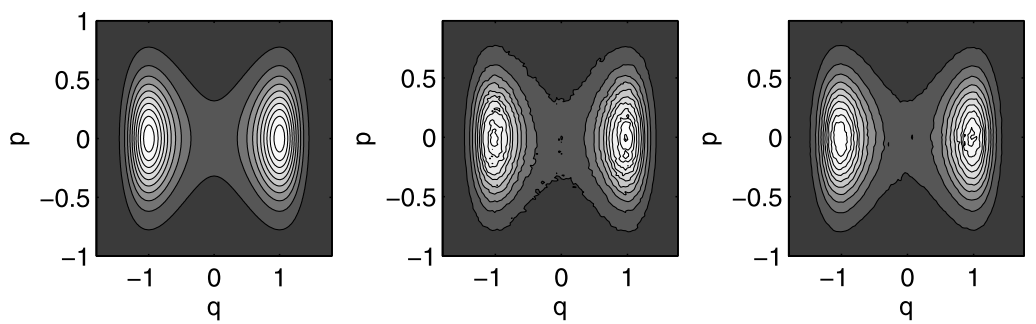

Fig. 1 Contour plot of the canonical equilibrium measure for a particle in a double well potential. Left: theoretical measure (23), middle: empirical measure calculated from a simulation of the NHL model (2) with $\epsilon=1$, right: empirical measure calculated from a simulation of the reduced Langevin model (21)

where $\Gamma(p)=2(\alpha \sigma)^{-2}\left(\beta p^{T} M^{-1} p-(d+1)\right) p$. The variables $W_{n}, W_{n+1 / 2}$, etc. in $(22 \mathrm{~b})-$ (22c) denote successive values of a Wiener process, i.e., the increments $W_{n+1 / 2}-W_{n}$ are drawn from a normal distribution with variance $\Delta t / 2$. The split-step backward Euler method (22a)-(22b) is implicit in the momenta, which for the simple example we consider is computationally feasible.

Numerical experiments were done for the following double well potential

$$
V(q)=\frac{1}{4} q^{4}-\frac{1}{2} q^{2}
$$

with $M=1$. In all experiments we took as initial conditions $\left(q_{0}, p_{0}\right)=(1,1 / 4)$ and set $\beta=10$ as the inverse temperature. In this case, the canonical equilibrium density of the system (20) is given by

$$
\rho(q, p)=\exp \left[-\beta\left(\frac{p^{2}}{2}+\frac{q^{4}}{4}-\frac{q^{2}}{2}\right)\right] .
$$

Figure 1 demonstrates that the NHL model (2) and the reduced Langevin model (21) both sample the canonical equilibrium measure, as designed. The canonical equilibrium measure (23) is shown on the left, along with nearly-converged empirical measures obtained from a single, long simulation each of the NHL model (2) and the reduced Langevin model (21), respectively, in the middle and on the right. Each simulation was run on the interval $t=\left[0,10^{5}\right]$ with $\Delta t=0.001$. If the time interval is increased by a factor 10 , the sampled empirical densities are indistinguishable from the theoretical density (23). The agreement is expected, by construction of the models, if the simulation is ergodic.

We next present results of simulations of the NHL model (2) for varying values of $\epsilon$. Recall that the variance of the thermostat variable $y$ is $\epsilon^{-2}$. We thus expect that for large values of $\epsilon$ the effect of the NHL thermostat will be weak on the chosen finite time interval $t \in\left[0,10^{3}\right]$, and the trajectories will behave nearly deterministically. The reduced Langevin model (18) is derived in the limit $\epsilon \rightarrow 0$, so we expect that the NHL dynamics will be more erratic in this limit. This behavior is confirmed by Figs. 2 and 3. For $\epsilon=100$ the trajectories are smooth and nearly periodic. The left potential well is only sampled once on this time interval. Note, however, that the NHL model satisfies the Hörmander condition [15] and is expected to be ergodic for this problem. Indeed, for a simulation on the much longer interval $t \in\left[0,10^{7}\right]$ the sampled measure is indistinguishable from the theoretical measure shown in Fig. 1 (left) for all values of $\epsilon$. For $\epsilon=10$ and $\epsilon=1$, the solution is still quite smooth but both wells are frequently visited on the given time interval. For $\epsilon=0.1$ the phase trajectories are much more erratic, with large fluctuations in the momentum. In this regime 

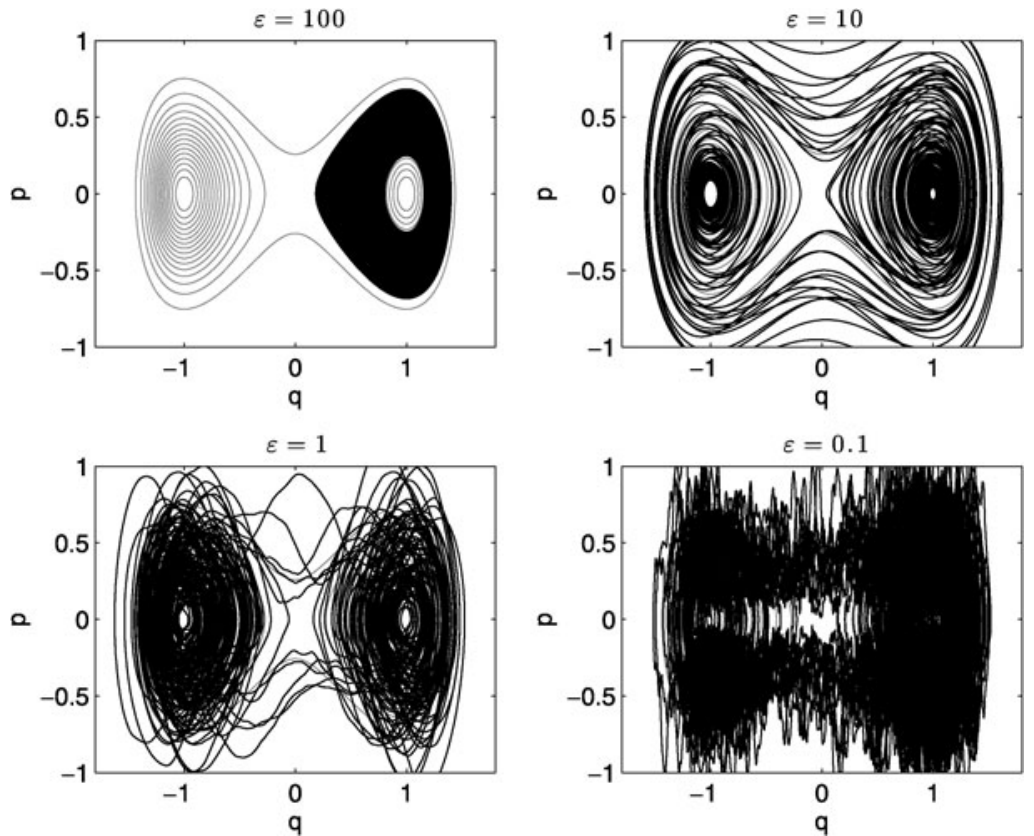

Fig. 2 Phase space trajectories for diminishing $\epsilon$, computed with the NHL model (2) using an integration stepsize of $\Delta t=10^{-3}$
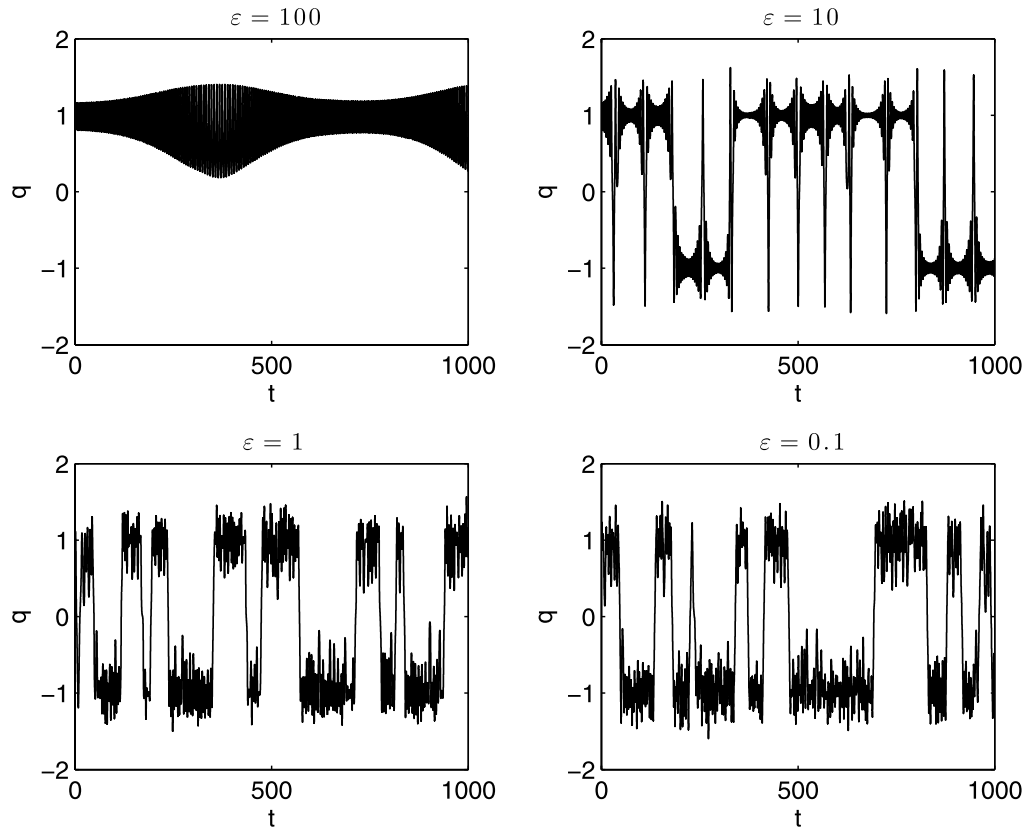

Fig. 3 Time series of position $q$ for diminishing $\epsilon$ with the NHL model (2). The integration stepsize is $\Delta t=10^{-3}$ 
Fig. 4 Log- $\log$ plot of the supremum error (24) on $t \in[0,10]$ between the numerical solution computed with the NHL dynamics (2) and that computed with the reduced Langevin model (21). A time step of $\Delta t=10^{-6}$ was used and identical Wiener increments have been employed for both models. The slope of the dashed line indicates quadratic convergence in $\epsilon$

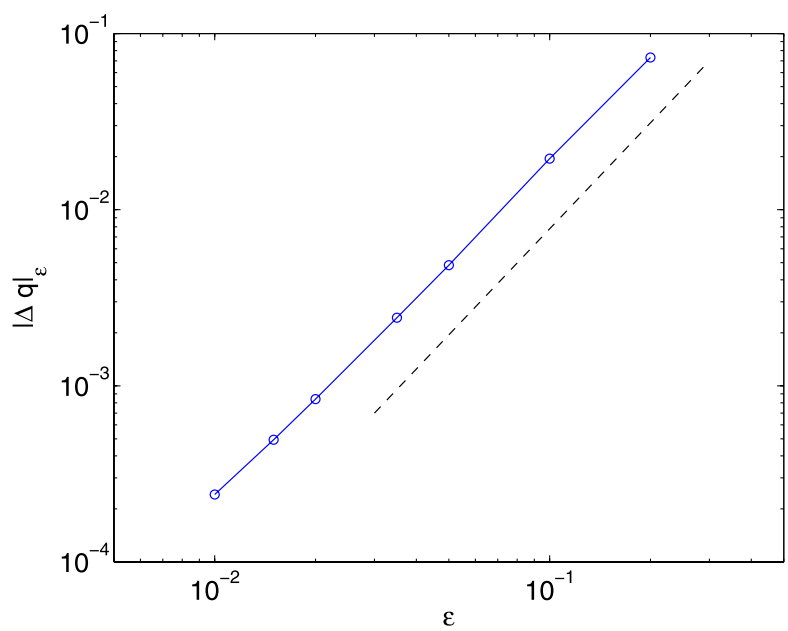

the trajectories look very similar to those of the Langevin model (18) (not shown). On the other hand, Fig. 3 shows that the sampling behavior of the double well potential is qualitatively similar for $\epsilon=1$ and $\epsilon=0.1$, suggesting that the time series for $q$ is approximately converged even for $\epsilon=1$. We can quantify the convergence by numerically estimating the mean residence time $\tau$, i.e. the average time the trajectory spends in the respective potential wells. We find that the mean residence times for $\epsilon=1$ and $\epsilon=0.1$, which are $\tau=101$ and $\tau=102$ respectively, are both close to the mean residence time of the reduced Langevin model with $\tau=103$. Whereas the dynamics of the position variable $q$ of the NHL model (2) has converged in the sense that its statistics converges to the statistics of the reduced model (18) for $\epsilon=1$, this is not the case for the momentum variable $p$. In Fig. 2 it is clearly seen that the variance of the momentum variable $p$ strongly differs for $\epsilon=1$ and $\epsilon=0.1$. We conclude that the small- $\epsilon$ limit corresponds to large stochastic forcing and large influence of the thermostat variable $y$, consistent with the scaling we proposed in the derivation of the reduced Langevin equation (18)-(19) from the NHL model (2).

We now show that trajectories of the reduced Langevin equation (18)-(19) converge pathwise to solutions of the full NHL model (2) in the limit of $\epsilon \rightarrow 0$. To study convergence we introduce the supremum error

$$
|\Delta q|_{\epsilon}=\sup _{t \in\left[0, t^{\star}\right]}\left|q^{\mathrm{NHL}}(t)-q^{\mathrm{red}}(t)\right|,
$$

between solutions of the full NHL model (2), denoted by $q^{\mathrm{NHL}}$, and solutions of the reduced Langevin model (21), denoted by $q^{\text {red }}(t)$, on a fixed time interval $\left[0, t^{\star}\right]$. To investigate pathwise convergence we use identical Wiener increments for both the NHL model (2) and the reduced Langevin model (21). How the error $|\Delta q|_{\epsilon}$ scales with $\epsilon$ is illustrated in Fig. 4 where a clear quadratic scaling is seen with $|\Delta q|_{\epsilon} \sim \epsilon^{-2}$. Although we do expect a scaling with $\epsilon$, we are not aware of any rigorous results that explain the quadratic behavior.

It is remarkable that the limiting Langevin process that we derive here by means of a stochastic singular perturbation analysis [19] has been heuristically proposed before e.g. by Leimkuhler et al. [12] and Bussi et al. [2, 3] to construct a thermostat with a mild effect on the dynamics, only along the direction of motion. 
Acknowledgements We are grateful to the Isaac Newton Institute where parts of this research were performed during the programme Mathematical and Statistical Approaches to Climate Modelling and Prediction (CLP). GAG acknowledges support from the Australian Research Council. JEF acknowledges support from the Netherlands Organization for Scientific Research (NWO).

\section{References}

1. Bulgac, A., Kusnezov, D.: Canonical ensemble averages from pseudomicrocanonical dynamics. Phys. Rev. A 42, 5045-5048 (1990)

2. Bussi, G., Parinello, M.: Stochastic thermostats: comparison of local and global schemes. Comput. Phys. Commun. 179, 26-29 (2008)

3. Bussi, G., Donadio, D., Parinello, M.: Canonical sampling through velocity rescaling. J. Chem. Phys. 126, 014101 (2007)

4. Dubinkina, S., Frank, J., Leimkuhler, B.: Simplified modelling of a thermal bath, with application to a fluid vortex system. SIAM Multiscale Model. Simul. 8, 1882-1901 (2010)

5. Higham, D.J., Mao, X., Stuart, A.M.: Strong convergence of Euler-type methods for nonlinear stochastic differential equations. SIAM J. Numer. Anal. 40, 1041-1063 (2002)

6. Hoover, W.: Canonical dynamics: equilibrium phase space distributions. Phys. Rev. A 31, 1695-1697 (1985)

7. Khasminsky, R.Z.: On stochastic processes defined by differential equations with a small parameter. Theory Probab. Appl. 11, 211-228 (1966)

8. Kurtz, T.G.: A limit theorem for perturbed operator semigroups with applications to random evolutions. J. Funct. Anal. 12, 55-67 (1973)

9. Kurtz, T.G.: Limit theorems and diffusion approximations for density dependent Markov chains. Math. Program. Stud. 5, 67-78 (1976)

10. Kurtz, T.G.: Strong approximation theorems for density dependent Markov chains. Stoch. Process. Appl. 6, 223-240 (1978)

11. Leimkuhler, B., Noorizadeh, E., Theil, F.: A gentle stochastic thermostat for molecular dynamics. J. Stat. Phys. 135, 261-277 (2009)

12. Leimkuhler, B., Noorizadeh, E., Penrose, O.: Comparing the efficiencies of stochastic isothermal molecular dynamics methods. J. Stat. Phys. (2011, to appear)

13. Legoll, F., Luskin, M., Moeckel, R.: Non-ergodicity of Nosé-Hoover thermostatted harmonic oscillator. Arch. Ration. Mech. Anal. 184, 449-463 (2007)

14. Legoll, F., Luskin, M., Moeckel, R.: Non-ergodicity of Nosé-Hoover dynamics. Nonlinearity 22, 16731694 (2009)

15. Mattingly, J.C., Stuart, A.M., Higham, D.J.: Ergodicity for SDEs and approximations: locally Lipschitz vector fields and degenerate noise. Stoch. Process. Appl. 101, 185-232 (2002)

16. Nosé, S.: A molecular dynamics methods for simulations in the canonical ensemble. Mol. Phys. 52, 255-268 (1984)

17. Nosé, S.: A unified formulation of the constant temperature molecular dynamics method. J. Chem. Phys. 81, 511-519 (1984)

18. Papanicolaou, G.C.: Introduction to the asymptotic analysis of stochastic equations. In: DiPrima, R.C. (ed.) Modern Modeling of Continuum Phenomena. AMS, Providence (1974)

19. Pavliotis, G.A., Stuart, A.M.: Multiscale Methods-Averaging and Homogenization. Texts in Applied Mathematics, vol. 53. Springer, New York (2008)

20. Samoletov, A., Chaplain, M.A.J., Dettmann, C.P.: Thermostats for "slow" configurational modes. J. Stat. Phys. 128, 1321-1336 (2007)

21. Givon, D., Kupferman, R., Stuart, A.: Extracting macroscopic dynamics: model problems and algorithms. Nonlinearity 17, R55-R127 (2004) 